\title{
Formative Evaluation of Data-Driven Business Models - The Data Insight Generator
}

\author{
Babett Kühne \\ University of Hamburg \\ babett.kuehne@uni-hamburg.de
}

\author{
Tilo Böhmann \\ University of Hamburg \\ tilo.boehmann@uni-hamburg.de
}

\begin{abstract}
New technological developments such as Big Data or, the Internet of Things lead to exponentially increasing amounts of data created and stored by organizations. As a consequence, new data-driven business models (DDBMs) appear. These business models have special characteristics which need to be included in the business model development process. Thus, different methods and tools have emerged to support the development of DDBMs. One of these is the Data Insight Generator (DIG) which seeks to combine the key resource and value proposition of a DDBM. This paper comprises the application of the thinking-aloud method for a formative evaluation of the DIG. The contribution of this paper is twofold. First, the usability of the DIG is tested and implications for further development are derived. Second, the paper provides empirically-based insights into development of DDBM that facilitate the future development of such business models.
\end{abstract}

\section{Introduction}

The strategic importance of information technologies is growing, which inspires more and more business innovations [1]. Organizations have increasingly turned from product-based offerings to service-oriented value propositions [2]. Such serviceoriented business models often rely on data-driven services [3] as organizational data sets are growing exponentially. The effective use of data is thus becoming a key factor for the survival of businesses [4]. Data and its analysis can generate new knowledge which could be served as a new value proposition for the customer [5]. Further, relationships with customers could be optimized with the analysis of data [1]. As a result, emerging data sets have influenced the improvement of efficiency in organizations [6].

As the design of a data-driven business models (DDBM) is not trivial, a business model representation can be used as a strategic tool to design such a business model [7]. In general, there is no lack of artifacts for the development and representation of business models. Many of these are designed within the field of research from which they originate [8]. As a result, there are a number of specialized artifacts in the research field.

The field of DDBM is relatively new [9]. There are specific characteristics of data-driven business models and the resulting design principles for their representation $[10,11]$. As a consequence, some specific representations for DDBM, like Data Canvas [12] and a canvas for data-driven ideation workshops [13] were developed. As the connection between data and value proposition in a DDBM plays an important role [14], we focus on this connection. The Data Insight Generator (DIG) [14] seeks for an illustration between these two components, key resources and value proposition [15], of a DDBM. This paper reports on the formative evaluation of this specific representation of DDBM.

Our formative evaluation of the DIG is based on the thinking-aloud method [16]. The evaluation is part of an iterative evaluation of the Design Science Research Process, in order to observe areas of improvement and enhancement during the artifact development phase [17]. As evaluation studies are relatively rare in DDBM research, our evaluation also creates general research interest in business model design. However, most business model representations are evaluated by illustrating a use case [18-20], but some exceptions exist. For example, the business model representation Resource Even Agent [21] was evaluated with an experiment of more than 100 business administration and information systems students [22]. Further, Zolnowski and Böhmann [23] also evaluated their business model representation Service Business Model Canvas with the thinkingaloud method [16]. Hence, this paper answers the following research question:

RQ: "Can the Data Insight Generator provide support for developing, understanding, and analyzing $D D B M ? "$ 


\section{Theoretical Foundations}

\subsection{Data-Driven Business Models (DDBM)}

The term business model has multiple definitions and bases for understanding [24, 25]. However, there are some common characteristics among these definitions. One key element of a business model is the value creation for the customer [26] and key components are business activities and inputs [24]. Resulting from these different definitions of a business model, a number of varying representations exist $[18$, $25,27]$. For example, some common meta models are Business Model Canvas [15] based on the Business Model Ontology [19], and e3-value [20], which contain different characteristics of a business model. The Business Model Canvas (BMC) is one prominent strategic management template [28]. It has nine building blocks that include value proposition, customers, finances and infrastructure [27]. The e3value shows an inter-organizational network of different actors which create, distribute and consume value together [20]. However, it is recommended that business model representations should be inspected in detail and validated. Also, further development of such representations is necessary [29].

As the BMC is one of most frequently used strategic management templates [28], extensions of it have been proposed over the last several years. One example is the Service Business Model Canvas (SBMC) [30] which illustrates a service-based business model [31]. There are different options to extend the BMC: (1) divide existing canvas fields; (2) modify canvas field content; (3) change the position of the fields; (4) add new fields and (5) link elements in the fields [32]. Further, a two-layered model could be integrated into a canvas field which consists of higherand lower-level elements [33].

Based on the service-oriented paradigm [34], new services appear in science as well as in praxis; these are called "Data-as-a-Service" or "Analytics-as-aService" [8, 24, 35]. DDBMs form a further development of these business models. Data sets are the key resource of DDBM [24]. However, there is no defined data threshold when comparing traditional business models with DDBMs [36]. Implementing data as a focus in the business model can have effects on value proposition, value creation and value capturing [36]. Thus, the transformation from a product-based business model to a service-orientated offering can be influenced by data-driven innovations [37, 38]. All in all, there are few guidelines in practice as well as in literature which can be used for development of a business model applying resource data in an effective way [39].

There are some artifacts which cover parts of a DDBM. With the Data Canvas, an organization can organize their data into four different canvas fields. These are differentiated by the source of data (internal or external) and the tonus of the data [12]. This artifact is only affecting the canvas field key resources of the BMC. The canvas for data-driven ideation workshops [13] integrates the design thinking method [40] into the development of a data-driven innovation. This canvas aims at developing new user-centric, data-driven ideas and is not intended to cover the generation of a full business model. There are two further artifacts which support the development of a single element of a DDBM. The first is a key activities tool which is focused on all the important key activities in a DDBM [41]. The second is a model which supports the cost benefit analysis of a DDBM [42]. Thus, this model is focused on the financial part of the analysis. As this paper addresses on the business model of data-driven innovations, we chose to analyze the Data Insight Generator (DIG) [14]. As described in the following section, a key challenge during the development of DDBMs is to make the use and value of data resources clear and transparent to all stakeholders. As a consequence, the artifact is directed at the key resources and value proposition of the business model and should be used as an addition to the BMC for DDBM. The DIG is introduced in the next section in more detail.

\subsection{Data Insight Generator (DIG)}

The artifact Data Insight Generator is introduced as a boundary object which supports communication between different stakeholder as a visualization tool [43]. The artifact was developed based on semistructured interviews and a literature review [14]. As identified, one of the main problem [44] with DDBM is that the purpose of the data use should be clear to all stakeholders [14]. The BMC does not explain this connection. Due to this, the DIG was developed as an extension of the BMC which can be used in addition to the BMC. The DIG connects the key resource data with the value proposition in order to make the purpose of the data usage clear [14]. The DIG can be used as a working tool between data scientists and specialized business departments and could further be used to present an idea to different stakeholders. Users can work with the DIG in their organization if they have an initial idea of a new DDBM [14].

Figure 1 shows the DIG that consists of vertical and horizontal elements. The DIG should be used in a workshop setting to work on one idea and 
communicate it afterward to additional stakeholders [14].

As the DIG is an iterative tool, the horizontal lines reflect this intention. The three lines Think, Validate and Know should be used during an iterative process. First, workshop participants gather everything on sticky-notes [44] in the Think row. Afterward, they sort these ideas into Validate or Know lines. If they are unsure about one fact, they use the Validate row and take a to-do list with them on how to validate that fact before the next iteration cycle. If everything is clear and participants are sure about this fact, it will be placed into the Know line. After a number of iteration cycles, participants should be able to sort everything into the Know row to show the idea could work in a business model context [14].

The horizontal lines should link the key resource data and the value proposition of a DDBM. It is possible to start on both sides of the DIG. (1) Participants can start with the data and try to find a new value proposition with the DIG. (2) Participants can start with a more problem-centric view and work to find a value proposition for a specific problem of the customer first. In this case, they would be using the DIG from right to left and end with the data which they need for their value proposition. The columns between Data and Value proposition should link these two [14].

As data sets are the main resource of a DDBM [36], the field data display all relevant information. At the beginning of the workshops, participants could collect several data sources. If some are not needed at the end of the process, they could be withdrawn. The second column is about the data quality which contains confidentiality, integrity and availability [45]. The third column concentrates on the combination of data and the type of needed infrastructure for combining it. With the fourth column, Analytics, tools and methods are stated which can analyze the data [46]. The result of these analytics is displayed in the fifth column (Insight). This shows which new information could be generated out of the data. Finally, the Value column displays the value proposition for the customer. The value proposition is based on the new insight the organization is creating with their analytics activities [14].

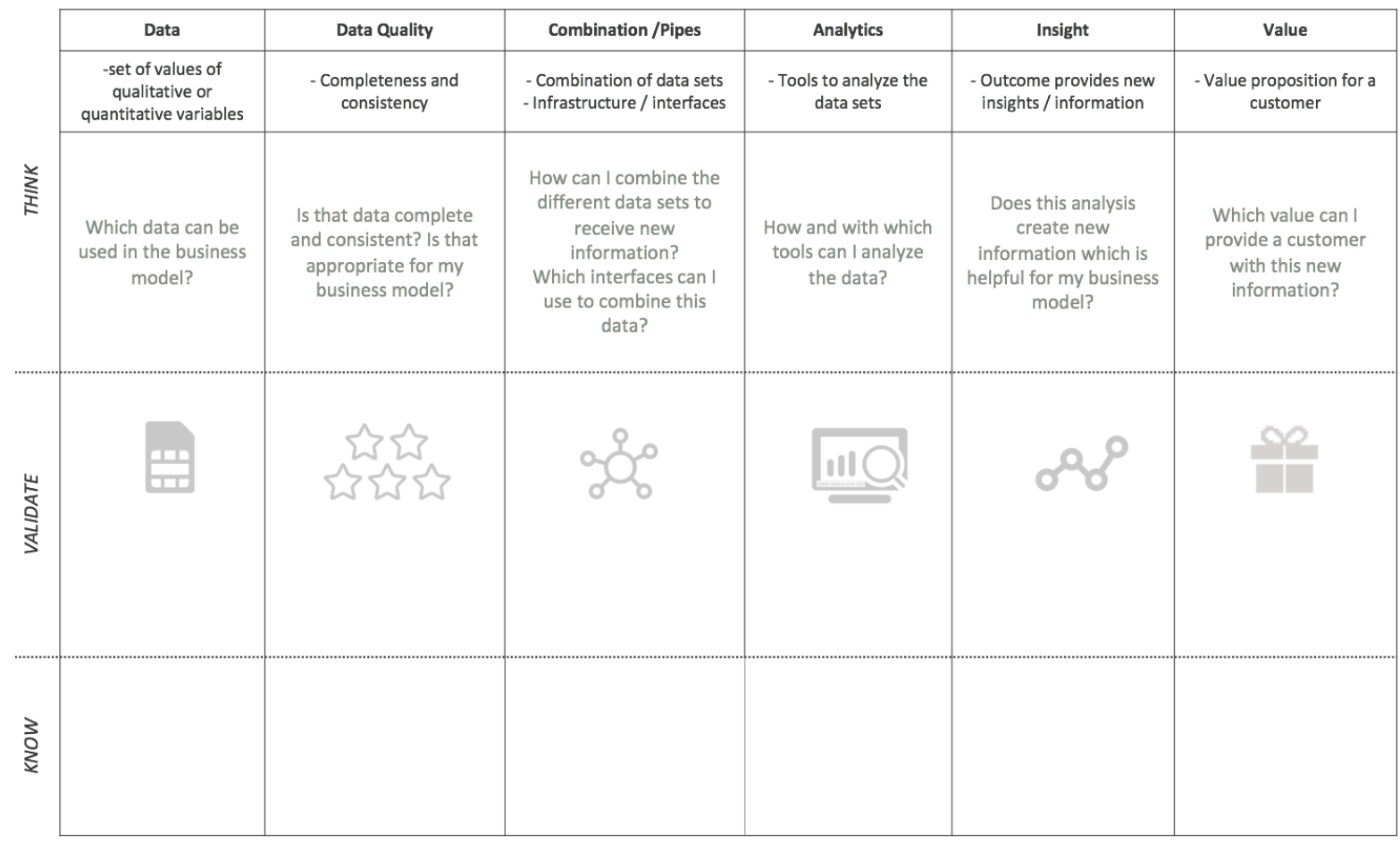

Figure 1: Data Insight Generator Version 1 [14]

\section{Methodology}

We used the Design Science Research Methodology (DSRM) [47] for this project. It is part of one evaluation cycle, which first evaluates an existing artifact, the DIG. Based on the evaluation results, we went back to the first phases of DSRM and redesigned the artifact. The improved version of the DIG may be found in section 4.3. The next section explains our research method in detail. 


\subsection{Thinking-Aloud Method}

As business model representations are boundary objects [43], they offer an illustration - graphical or textual - and improve the practical work with regard to business models. To ensure this, a business model representation needs to consist of easily understandable constructs and structural elements. As the DIG is such a tool, we chose to facilitate the development of DDBM by exploring how people in an organization understand and use the DIG. In order to do so, we used the thinking-aloud method which is an empirical evaluation for design methods [48].

The thinking-aloud method involves analysis of how the user considers the application of an artifact [49]. As it is based on the area of cognitive psychology, it is used widely in research to analyze human behavior. Also in the information systems field, this method is used for usability testing [50]. However, the method could be proceeded by different goals related to diverse research areas. For example, this could focus on humans' cognitive processes, on the one hand. On the other hand, the usability of a system which is under development could be tested as well. Normally, the thinking-aloud method is used in an advanced stage of the development process for usability testing [51].

The thinking-aloud method is closely linked to interviewees' cognitive processes without influencing those processes. It is a verbal report from the interviewee who is asked to articulate thoughts regarding using the artifact. In doing so, there are some rules which need to be followed in order to preserve high reliability of this method. For this study, we first collected only hard verbal data. These related each activity the interviewee attended. Data relating to topics such as inference, introspection and opinion were not included. Second, we provided detailed instructions before implementing use of the thinking-aloud method. We did so because it was necessary for the interviewee to be able to talk fluently without interruption. Third, we reminded the participants to keep talking if they held a pause than 20 seconds during the thinking-aloud test. Fourth, we did not intervene with the user by giving help or anything else. In doing so, we only reminded them to talk sometimes and did not interact in any way with the participants $[49,50]$.

\subsection{Participants}

This project involved 12 participants from research as well as practice. An overview about the participants is shown in Table 1. As $10+/-2$ participants [51] are necessary to detect $80 \%$ of usability problems overall, we chose this number of participants. We decided to recruit a mix from practice and research. Seven thinking-aloud protocols were conducted with experts from practice and five tests were conducted with researchers from different fields. In order to detect as many usability problems as possible, we conducted interviews with participants from various industries such as logistics, finance and the aircraft industry. All industry participants are considered experts in the field of DDBM. As they are from separate industries and have different roles in their organizations, they provided varying perspectives on the thinking-aloud protocols. The participants from other industries, including academia, were not familiar with the DIG at all. In doing so, we were careful not to taint the thinking-aloud protocols.

Table 1: Overview of Participants

\begin{tabular}{|l|l|l|}
\hline $\begin{array}{l}\text { Number of } \\
\text { Participants }\end{array}$ & Industry & Role \\
\hline 1 & Finance & Head of data analytics \\
\hline 1 & Insurance & CIO assistant \\
\hline 1 & Technology & Product owner \\
\hline 1 & Consulting & Consultant \\
\hline 1 & E-commerce & Agile coach \\
\hline 1 & Aircraft & Head of digital products \\
\hline 1 & Logistics & Demand manager \\
\hline 5 & University & Researchers \\
\hline
\end{tabular}

\subsection{Research Design}

To ensure the results were comparable with each other, we established a standardized setting for each thinking-aloud test. All sessions were conducted in a separate room so that participants would not be disturbed. Further, we monitored each session and reminded participants to keep talking. As we standardized the process, we began with a recorded audio sequence which contained an explanation of the DIG, the rules of the thinking-aloud test, and a reminder to keep talking. Before the audio was played, the participants received two sheets of paper. The first one contained the DIG as shown in Figure 1. The second sheet contained a use case description and the tasks of the thinking-aloud test.

After the audio was played, the participants started to read the use case. Afterward, they started to execute the thinking-aloud test by answering the questions mentioned on the second sheet of paper they received. As recommended by the literature, we used a neutral encouraging sound to remind the participants to keep talking, if they stopped talking for more than 20 seconds [50]. We recorded each thinking-aloud test with an audio recorder. 
We choose a use case which was identifiable for each participant as all of them have different backgrounds. The use case was about a new datadriven service that promised a less stressful start to the day for a customer whose car has an ice layer on the windshield. The service is combined with an alarm sent to the customer's phone which should wake the customer up five minutes earlier if there is an ice layer on the car. The use case gives more information as background. First, the basic data are provided by a temperature sensor in the car. Second, the interface which transfers the data to the customers' mobile phone is a Bluetooth interface. The text gives also the hint that this interface could be not reliable if the phone and the car are too far away from each other. Third, there is an application for the mobile phone available which was developed by an external organization.

In addition to the thinking-aloud method, we added a short semi-structured interview part [52] in which we asked general feedback questions about the DIG. The questions were raised depending on what the interviewee stated before. Thus, we could receive more information from the interviewees than in a structured interview. In all, we integrated at least four questions in the interview. First, we asked the participants if they would use the DIG in their organization. Second, we asked for feedback about unclear points or questions about the DIG. Third, the interviewees were asked to state if they would see the DIG as a communication tool (boundary object) between different stakeholder groups. Fourth, the interviewees were asked to mention the target audience of the DIG.

\subsection{Instructions}

In order to perform a formative evaluation of the DIG, we formulated seven tasks. All of these tasks represented problem-solving activities which are generally used for the thinking-aloud method [49, 53]. We designed two kinds of tasks which were differentiated by complexity [54]. The first four tasks had a lower complexity than the last ones. The participants had to conduct tasks which integrated the use case in the DIG. Thus, these tasks reflected their understanding of the use case and the overall DIG. Second, the last tasks were more complex because the participants needed to change something in the DIG. This showed, the degree of detail to which participants understood the DIG. In doing so, we aimed to evaluate adaptation of the DIG.

Participants were asked the following:

1.) Please use the DIG to classify the data sources.
2.) Could you use the DIG to evaluate the data quality?

3.) How can you visualize the mentioned data combinations and interfaces?

4.) Please fill out the canvas fields Analytics, Insight and Value fields for the example.

5.) What is the difference between the Insight and Value field?

6.) What would change in the DIG if there was a sensor in the windshield that could detect an ice layer?

7.) What would change in the DIG if you could use a WLAN interface from the car?

\subsection{Data Analysis}

After execution of each design-thinking test, we transcribed each one in a thinking-aloud protocol. Based on these transcripts, we analyzed the understanding, use, and navigation of the DIG. The aim of doing so was to understand and analyze utilization of the DIG and detect usability problems during DIG's application.

As we focused on these aims, we differentiated between problem types. According to the literature, five different problem types can be differentiated during a thinking-aloud test [55]. Thus, these problem types could be layout problems, data entry problems, feedback problems, terminology problems, and comprehensiveness problems. As the DIG is not an information technology artifact that gives direct feedback to the user, we excluded the feedback problems from our analysis.

Layout problems occur if a participant cannot find an element of the DIG within it. Terminology problems occur if some terms are not understood correctly and the participant uses them in another context. Question five especially focuses on these problems. Data entry problems appear if the participant does not know where to put data elements in the representation. Comprehensiveness problems point out that there is some missing information which is relevant to using the DIG in the right way [55].

In addition, we measured the time which was needed to perform the tasks in order to evaluate performance. Further, we considered whether participants were able to complete the tasks properly. The results of our thinking-aloud tests are described in the next section.

\section{Results}

\subsection{Task Performance}


The average duration of the thinking-aloud tests was 8 minutes and 48 seconds. The longest session took 13 minutes and 55 seconds, and the shortest took 4 minutes and 58 seconds. The large difference between some interviews could be explained because some of the interviewees added additional ideas which were not given in the text. There was no difference between practitioners and researchers regarding the needed time and the detected problems. We only could recognize that participants without knowledge in DDBM took less time because they did not find any other ideas and only passed the tasks as requested, which supported that the tool should be used in a workshop setting which contains both a data scientist and participants with a deep knowledge of the business.

Table 2 shows an overview about the duration of the tasks and whether they were completed successfully.

Table 2: Task performance

\begin{tabular}{|c|c|c|c|c|}
\hline Task & $\begin{array}{l}\text { Number of } \\
\text { participants } \\
\text { who completed } \\
\text { the task } \\
\text { properly }\end{array}$ & Mean & Median & $\begin{array}{l}\text { Standard } \\
\text { deviation }\end{array}$ \\
\hline 1 & 12 & $\begin{array}{l}1 \min 8 \\
\sec \end{array}$ & $\begin{array}{l}1 \min 3 \\
\sec \end{array}$ & $45 \mathrm{sec}$ \\
\hline 2 & 11 & $58 \mathrm{sec}$ & $\begin{array}{l}1 \min 3 \\
\sec \end{array}$ & $34 \mathrm{sec}$ \\
\hline 3 & 8 & $\begin{array}{l}1 \mathrm{~min} \\
11 \mathrm{sec}\end{array}$ & $\begin{array}{l}1 \min 6 \\
\sec \end{array}$ & $32 \mathrm{sec}$ \\
\hline 4 & 8 & $\begin{array}{l}2 \mathrm{~min} \\
28 \mathrm{sec}\end{array}$ & $\begin{array}{l}2 \min 15 \\
\mathrm{sec}\end{array}$ & $57 \mathrm{sec}$ \\
\hline 5 & 11 & $57 \mathrm{sec}$ & $50 \mathrm{sec}$ & $36 \mathrm{sec}$ \\
\hline 6 & 12 & $\begin{array}{l}1 \min 1 \\
\mathrm{sec}\end{array}$ & $55 \mathrm{sec}$ & $28 \mathrm{sec}$ \\
\hline 7 & 10 & $\begin{array}{l}1 \min 9 \\
\text { sec }\end{array}$ & $50 \mathrm{sec}$ & $56 \mathrm{sec}$ \\
\hline Sum & & $\begin{array}{l}8 \mathrm{~min} \\
48 \mathrm{sec}\end{array}$ & $\begin{array}{l}8 \mathrm{~min} 43 \\
\mathrm{sec}\end{array}$ & $\begin{array}{l}2 \min 46 \\
\sec \end{array}$ \\
\hline
\end{tabular}

As shown in the Table, most participants were able to complete the tasks properly during the application of the DIG. The result was satisfactory as most participants could complete the tasks properly. Question 5 was overseen by one participant; that is why only 11 participants could complete this task. Three participants, however, correctly explained the difference between the Insight and Value; they did not use it as expected with the use case. Further, problems appeared with the combination and pipes field due to some participants forgetting the combination or pipes.

The derivation of how long it took the participants to answer a question is different. Some interviewees go through the process rather quickly; some took a little bit longer. The ones who took longer did not have problems with the DIG itself. They went deeper into the example and found some elements which were not mentioned in the use case. For example, some participants found new data sources like weather data which is available online; others found new business model ideas which are related to the use case, like the use of a heat map for parking or a service which gives customers a reminder to put some protection on their windshield if there is a high chance of an ice layer forming overnight. Two participants a step further. They realized that the integration of new sensors would be more expensive than integrating more external data sources into the business model.

\subsection{Detected Problems}

In this section, we will introduce problems which occurred during the think-aloud test. As mentioned before, they will be differentiated among the categories of layout, terminology, data entry, and comprehensiveness problems.

In summary, we identified seven participants who had no problems solving all tasks properly. One participant was not able to answer the question about data quality because of individual problems with the use case example. Of the participants, $33.33 \%$, had problems with field Combination and pipes. Mainly, they did not realize this problem, but they used the field only for one aspect. One part of them only entered the combination of data sets; the other half only noted the infrastructure. One participant realized that he forgot Combinations at the end of the test. Another $33.33 \%$ of the participants had problems with the Analytics field. These problems occurred due to the fact that the use case does not gave any examples for analytics tools. Thus, some only used the given information in the use case. One participant who had problems with the Analytics field stated, "If I should fill out that for our department, I would know which tools and methods I would use in this case." As a result, the problem was issued by the example and not by using the DIG itself. Furthermore, these participants did not use the Insight field as it was intended. They used it as an internal perspective. In doing so, they noted some evaluation points in this field. Thus, they interpreted the Insight field as an insight about whether the business model was working. In contrast, all of them answered question five correctly. Thus, they understood this field in theory but did not apply it as intended. One participant forgot to read question five, which is why only 11 participants passed that without a problem. Two participants could not answer the last question. These problems occurred because they did 
not use the Combination and pipes line for the infrastructure part.

We detected layout problems mainly in the first tasks of our thinking-aloud test. As some of the participants forgot the combination or infrastructure side in the field Combination and pipes, we identified that there is a layout problem. Due to having two different aspects in one field, one of them was overlooked.

We also detected terminology problems in the canvas fields Insights and Value. The insight was seen in some cases as an internal perspective. From this perspective, participants used the field for a validation of the business model. Thus, they noted that the business model would not work due to data quality issues. In contrast, they were able to explain the Insight field as it was intended. One could argue that these problems occurred due to the given example which had data quality problems but recognizing that such data problems could also happen in a real-world situation. Thus, this problem could be solved with a clearer description in the canvas field. We additionally detected small problems with the Value field, wherein the participants needed to read the description and question carefully. The headline was too broad for quickly understanding this canvas field.

Some participants had problems entering the Bluetooth interface; this can be identified as a data entry problem. This problem is deeply linked with the layout problem of the canvas field Combination and pipes. The participants who did not recognize the Bluetooth interface already used the field Combination and pipes for the combination of given data sets.

We cannot indicate comprehensiveness problems in our thinking-aloud protocols. Thus, we cannot find any missing elements in the DIG. However, more elements would increase the complexity of the DIG which would make the application harder.

\subsection{Improved Data Insight Generator}

As stated in the last section, we identified two main problems, and one small one in the DIG.

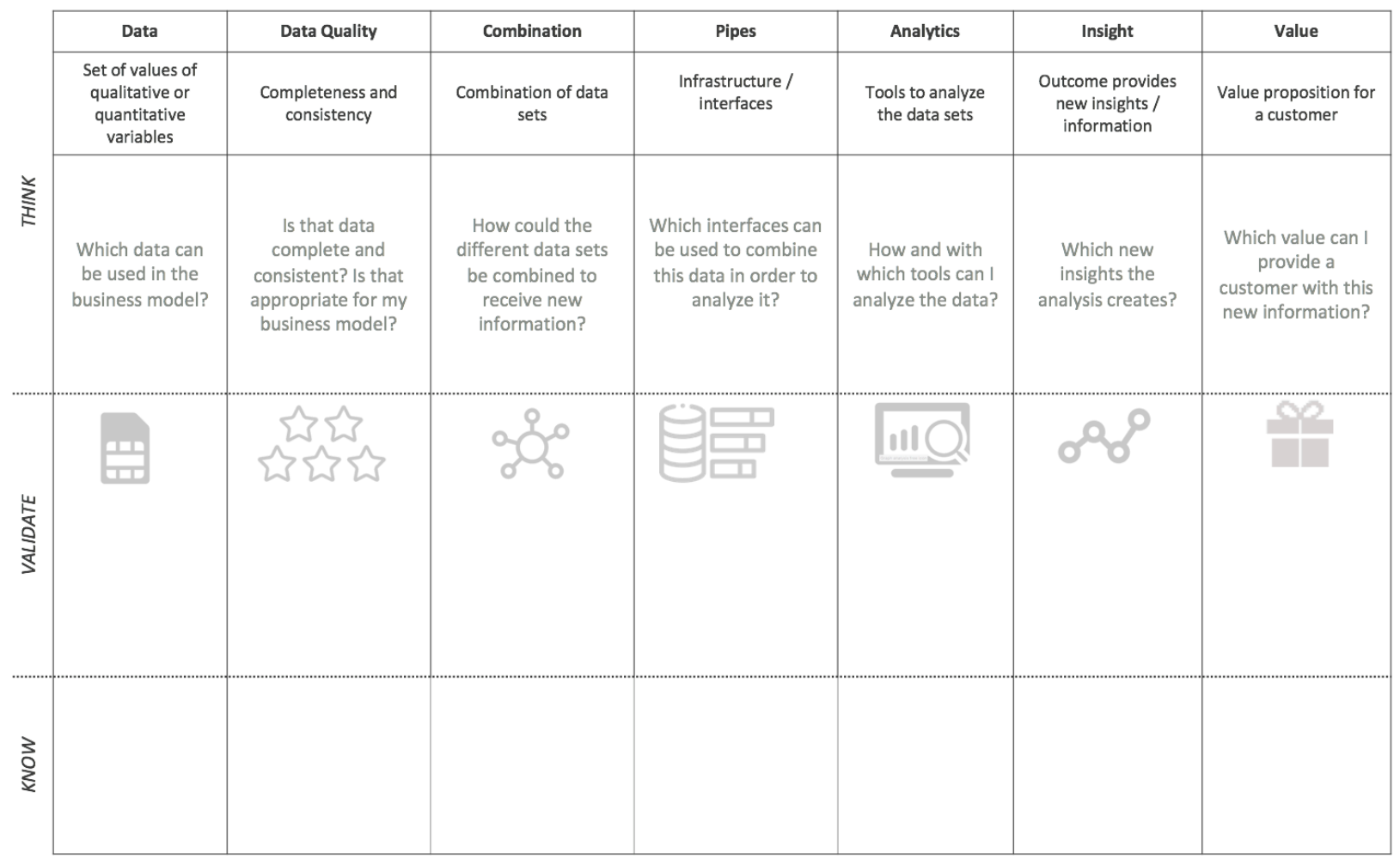

Figure 2: Data Insight Generator Version 2

First, we identified the layout problem with the combination and pipes. Some participants used this canvas field only for one, combination or pipes. As a result, we decided to split these fields. We decided to place the combination field after the data quality field and the pipes after that. We did this for two reasons. 
First, the combination is more related to the data and data quality fields on the left side of the canvas. Second, the participants, who solved the question about combination and pipes properly, started with combination and went to the interfaces after they combined the data.

Second, we decided to reframe the question in the insight field to make the intention clearer. Further, the question is now more open than in the first version of the DIG. We also changed the headline in the right canvas field from Value to Value Proposition in order to improve the understanding of this field and make it more consistent

\section{Discussion}

Our formative evaluation with the thinking-aloud protocols allowed us to use the information and develop an improved version of our artifact. Further, the interviews after the thinking-aloud test offered the opportunity to receive more feedback and useful information about the DIG.

All participants would use the DIG in their organization. This positive feedback gave us the insight that the DIG has practical contributions for practitioners. Thus, we can support practitioners in developing new data-driven business models in their organization. Further, the practitioners emphasized that they like the two possible ways through the DIG, one starting on the data side and one starting on the value proposition side.

As we already realized in the thinking-aloud protocols, four participants stated that the difference between combination and pipes was unclear to them. We fixed this issue dividing this canvas field into two different ones. Aside from that issue, the DIG was clear to all participants. Only one participant stated the improvement to add a data availability field into the canvas. However, data availability was integrated into the data field itself. Further, two participants underlined that the lines (Think, Validate and Know) are very useful and improve the application of the DIG. Moreover, participants emphasized that the differentiation between analytics, insights and value proposition improved their structured thinking because these three constructs are mixed with each other most of the times.

Mostly all participants saw the DIG as a communication tool between different stakeholders. Only one stated that he or she would use only the last two fields (Insight and Value Proposition) as a communication interface to other non-data scientist stakeholders. However, the different perspectives improved the understanding of how the value is created, e.g. stakeholder with different backgrounds could understand what additional data were needed or why the used data are needed. As such stakeholders can have different backgrounds, we also asked the participants about the target audience of the DIG. Mainly, they stated interdisciplinary groups as the target audience. Some also mentioned nonexperienced data scientists, information technology departments, information systems departments, business development professionals, product managers, product owners and requirements engineers as members of the target audience.

\section{Conclusion}

We conducted a formative evaluation of the DIG in this study. To do so, we adapted a thinking-aloud test [49]. We used this method because it originated in the area of cognitive psychology which is widely applied in usability testing. As the DIG is a boundary object which should apply a common understanding with different stakeholders, the thinking-aloud method matches as an analysis of human behavior. Based on our results of the thinking-aloud test, we improved the artifact of the DIG and deepened the knowledge of DDBM.

Our research provides these contributions. First, we indicate that a formative evaluation, such as a thinking-aloud test, can be used to evaluate boundary objects of business model representations. As the most business model evaluations use summative evaluation methods, we show that thinking-aloud tests could be also used for evaluation of boundary objects. Thus, we support the work of Zolnowski and Böhmann [23] who also performed a formative evaluation for a business model representation. Second, we deepen the knowledge about DDBMs by improving the constructs of the DIG. As we separated the canvas field Contribution and pipes, our research also emphasized that a canvas field in a boundary object should consist of only one construct. Third, we have shown the practical contribution of our research as the interviews stated that the DIG is a useful artifact and participants would apply the DIG in their organization.

However, our study has some limitations. First, we did not use a real-world use case. Thus, we did not choose a use case which was located in one of the participants' organizations. However, our chosen example was tangible for everyone, and they could project their thoughts onto the example. We chose a general example because of the different backgrounds of participants. Second, our study was short-term and formative. Nevertheless, our findings 
show that this kind of study yields new knowledge. However, we recommend adding a long-term and summative evaluation adapting the DIG as future research. Such a study could be done in a real-world situation and could yield improvement issues that did not appear in our study, yet.

In general, we show that boundary objects are a helpful construct for designing business models. As described in this study, it makes sense to integrate only one construct into one canvas field. As we did not find such design principles for boundary objects in the context of business model representations yet, we suggest a meta-study to explore such design principles for future research. Primary research about business modelling languages points researchers in the right direction $[28,44]$. Thus, we suggest adding the perspective of boundary objects to this research and defining design principles for designing a business model representation as a boundary object.

\section{References}

[1] V. Morabito, Big data and analytics. Strategic and organisational impacts. Heidelberg: Springer, 2015.

[2] N. Weiner and A. Weisbecker, "A business model framework for the design and evaluation of business models in the internet of services," in SRII Global Conference (SRII), 2011 Annual, 2011: IEEE, pp. 21-33.

[3] K. Williams, S. Chatterjee, and M. Rossi, "Design of emerging digital services: a taxonomy," European Journal of Information Systems, vol. 17, no. 5, pp. 505-517, 2008.

[4] J. Brownlow, M. Zaki, M. Neely, and F. Urmetzer, "Data and analytics-Data-driven business models: A blueprint for innovation," in "Cambridge Service Alliance," 2015.

[5] A. McAfee, E. Brynjolfsson, T. H. Davenport, D. Patil, and D. Barton, "Big data," The management revolution. Harvard Bus Rev, vol. 90, no. 10, pp. 61-67, 2012.

[6] R. Schüritz and G. Satzger, "Patterns of Data-Infused Business Model Innovation," in IEEE Conference on Business Informatics (CBI), Paris, France, 2016, vol. 1: IEEE, pp. 133-142.

[7] N. M. Bocken, S. W. Short, P. Rana, and S. Evans, "A literature and practice review to develop sustainable business model archetypes," Journal of cleaner production, vol. 65, pp. 42-56, 2014.

[8] F. Beha, A. Göritz, and T. Schildhauer, "Business Model Innovation: the Role of Different Types of Visualisations," in XXVI ISPIM Conference. Shaping Vrontiers of Innovation, 2015, pp. 1-19.

[9] P. M. Hartmann, M. Zaki, N. Feldmann, and A. Neely, "Big data for big business? A taxonomy of data-driven business models used by start-up firms," in "Cambridge Service Alliance," 2014.

[10] B. Kühne and T. Böhmann, "Requirements for Representing Data-Driven Business Models - Towards Extending the Business Model Canvas," in Twenty-fourth
Americas Conference on Information Systems, New Orleans, 2018, pp. 1-10.

[11] B. Kühne, A. Zolnowski, J. Bornholt, and T. Böhmann, "Making Data Tangible for Data-driven Innovations in a Business Model Context," in 25th American Conference on Information Systems, Cancun, Mexico., 2019, pp. 1-10.

[12] K. Mathis and F. Köbler, "Data-Need Fit-Towards Data-Driven Business Model Innovation," in Service Design Geographies. Proceedings of the ServDes. 2016 Conference, 2016, no. 125: Linköping University Electronic Press, pp. 458-467.

[13] T. Kronsbein and R. Mueller, "Data Thinking: A Canvas for Data-Driven Ideation Workshops," in Proceedings of the 52nd Hawaii International Conference on System Sciences, 2019.

[14] B. Kühne and T. Böhmann, "Data-Driven Business Models - Building the Bridge Between Data and Value," presented at the 27th European Conference on Information Systems, Stockholm, Sweden, 2019.

[15] A. Osterwalder and Y. Pigneur, Business model generation: a handbook for visionaries, game changers, and challengers. John Wiley \& Sons, 2010.

[16] K. A. Ericsson and H. A. Simon, "How to study thinking in everyday life: Contrasting think-aloud protocols with descriptions and explanations of thinking," Mind, Culture, and Activity, vol. 5, no. 3, pp. 178-186, 1998.

[17] J. Venable, J. Pries-Heje, and R. Baskerville, "A comprehensive framework for evaluation in design science research," in International Conference on Design Science Research in Information Systems, 2012: Springer, pp. 423438.

[18] M. M. Al-Debei and D. Avison, "Developing a unified framework of the business model concept," European Journal of Information Systems, vol. 19, no. 3, pp. 359-376, 2010.

[19] A. Osterwalder, "The business model ontology a proposition in a design science approach," Université de Lausanne, Faculté des hautes études commerciales, 2004.

[20] J. Gordijn, "E ${ }^{3}$-value in a Nutshell," in International Worksop on E-Business Modeling, HEC Business School, 2002: Citeseer.

[21] J. Buder and C. Felden, "Towards a reference model of business model \& business process management alignment," in 6th International Workshop on Value Modeling and Business Ontology. Vienna, Austria. BurtonJones A, Wand Y, and Weber $R$ (2009) Guidelines for Empirical Evaluations of Conceptual Modeling Grammars. Journal of the Association for Information Systems, 2012, vol. 10, no. 6, pp. 495-532.

[22] G. Poels, A. Maes, F. Gailly, and R. Paemeleire, "The pragmatic quality of Resources-Events-Agents diagrams: an experimental evaluation," Information Systems Journal, vol. 21, no. 1, pp. 63-89, 2011.

[23] A. Zolnowski and T. Böhmann, "Formative evaluation of business model representations-The service business model canvas," 2014.

[24] M. Sorrentino and M. Smarra, "The Term "Business Model" in Financial Reporting: Does It Need a Proper Definition?," Open Journal of Accounting, vol. 4, no. 02, p. $11,2015$. 
[25] C. Zott, R. Amit, and L. Massa, "The business model: recent developments and future research," Journal of management, vol. 37, no. 4, pp. 1019-1042, 2011.

[26] M. Lund and C. Nielsen, "The evolution of networkbased business models illustrated through the case study of an entrepreneurship project," Journal of Business Models, vol. 2, no. 1, pp. 105-121, 2014.

[27] A. Osterwalder, Y. Pigneur, and C. L. Tucci, "Clarifying business models: Origins, present, and future of the concept," Communications of the association for Information Systems, vol. 16, no. 1, p. 1, 2005.

[28] T. John, D. Kundisch, and D. Szopinski, "Visual languages for modeling business models: A critical review and future research directions," in 38rd International Conference on Information Systems, Seoul, 2017.

[29] D. Veit et al., "Business models," Business \& Information Systems Engineering, vol. 6, no. 1, pp. 45-53, 2014.

[30] A. Zolnowski, C. Weiss, and T. Böhmann, "Representing Service Business Models with the Service Business Model Canvas - The Case of a Mobile Payment Service in the Retail Industry," presented at the 47th Hawaii International Conference on System Sciences, Hawaii, 2014.

[31] A. Zolnowski and T. Böhmann, "Business modeling for services: Current state and research perspectives," presented at the Proceedings of the Seventeenth Americas Conference on Information Systems, Detroit, 2011.

[32] T. Schoormann, D. Behrens, E. Kolek, and R. Knackstedt, "Sustanability in Business Models - A Literature-Review-Based Design-Science-Oriented Research Agenda," in Twenty-Fourth European Conference on Information Systems (ECIS), Istanbul, 2016, pp. 1-16.

[33] E. Fielt, "Conceptualising business models: Definitions, frameworks and classifications," Journal of Business Models, vol. 1, no. 1, pp. 85-105, 2014.

[34] S. L. Vargo and R. F. Lusch, Service-dominant logic (Permises , Perspectives, Possibilities). Cambridge: Cambridge University Press, 2014.

[35] F. Hunke, C. Engel, R. Schüritz, and P. Ebel, "Understanding the Anatomy of Analytics-Based Services - a Taxonomyto Conceptualize the Use of Data and Analytics in Services," in Twenty-Seventh European Conference on Information Systems, Stockholm-Uppsala, Sweden, 2019.

[36] R. Schüritz, S. Seebacher, and R. Dorner, "Capturing Value from Data: Revenue Models for Data-Driven Services," in Proceedings of the 50th Hawaii International Conference on System Sciences, Hawaii, 2017, pp. 53485357.

[37] R. M. Schüritz, S. Seebacher, G. Satzger, and L. Schwarz, "Datatization as the Next Frontier of Servitization-Understanding the Challenges for Transforming Organizations," in Thirty Eighth International Conference on Information Systems, South Korea, 2017.

[38] C. Engel and P. Ebel, "Data-Driven Service Innovation: A Systematic Lliterature Rerview and Development of a Reserach Agenda," in 45th European Conference on Information Systems, Stockholm, 2019.
[39] T. John and D. Szopinski, "Towards Explaining the Popularity of the Business Model Canvas: A Dual Coding Approach," in Multikonferenz Wirtschaftsinformatik 2018, Lüneburg, 2018.

[40] T. Brown, "Design thinking," Harvard business review, vol. 86, no. 6, p. 84, 2008.

[41] F. Hunke and T. Wambsganß, "Turning Data into Value: Towards an Ideation Tool for Key Activities of Data-Driven Business Models," in 3rd Karlsruhe Service Summit Research Workshop, Karlsruhe, 2017.

[42] A. Zolnowski, J. Anke, and J. Gudat, "Towards a Cost-Benefit-Analysis of Data-Driven Business Models," presented at the International Conference on Wirtschaftsinformatik, St. Gallen, Switzerland, 2017.

[43] R. H. Von Alan, S. T. March, J. Park, and S. Ram, "Design science in information systems research," MIS quarterly, vol. 28, no. 1, pp. 75-105, 2004.

[44] H. Avdiji, D. Elikan, S. Missonier, and Y. Pigneur, "Designing tools for collectively solving Ill-structured problems," in Proceedings of the 51st Hawaii International Conference on System Sciences, 2018.

[45] M. Bulger, G. Taylor, and R. Schroeder, "Data-driven business models: challenges and opportunities of big data," ed: Oxford Internet Institute. Research Councils UK: NEMODE, New Economic Models in the Digital Economy, 2014.

[46] C. Dremel, S. Overhage, S. Schlauderer, and J. Wulf, "Towards a Capability Model for Big Data Analytics," presented at the International Conference on Wirtschaftsinformatik, St. Gallen, 2017.

[47] A. Hevner and S. Chatterjee, Design science research in information systems. Springer, 2010.

[48] K. Siau and M. Rossi, "Evaluation techniques for systems analysis and design modelling methods-a review and comparative analysis," Information Systems Journal, vol. 3, no. 21, pp. 249-268, 2011.

[49] K. A. Ericsson and H. A. Simon, Protocol analysis: Verbal reports as data. the MIT Press, 1984.

[50] T. Boren and J. Ramey, "Thinking aloud: Reconciling theory and practice," IEEE transactions on professional communication, vol. 43, no. 3, pp. 261-278, 2000.

[51] W. Hwang and G. Salvendy, "Number of people required for usability evaluation: the $10 \pm 2$ rule," Communications of the ACM, vol. 53, no. 5, pp. 130-133, 2010.

[52] E. Drever, Using Semi-Structured Interviews in SmallScale Research. A Teacher's Guide. ERIC, 1995.

[53] J. E. Russo, E. J. Johnson, and D. L. Stephens, "The validity of verbal protocols," Memory \& cognition, vol. 17, no. 6, pp. 759-769, 1989.

[54] Z. Guan, S. Lee, E. Cuddihy, and J. Ramey, "The validity of the stimulated retrospective think-aloud method as measured by eye tracking," in Proceedings of the SIGCHI conference on Human Factors in computing systems, 2006: ACM, pp. 1253-1262.

[55] M. Van Den Haak, M. De Jong, and P. Jan Schellens, "Retrospective vs. concurrent think-aloud protocols: testing the usability of an online library catalogue," Behaviour \& information technology, vol. 22, no. 5, pp. 339-351, 2003. 